\title{
Engineering a Highly Scalable Object-aware Process Management Engine Using Distributed Microservices
}

\author{
Kevin Andrews, Sebastian Steinau, and Manfred Reichert \\ Institute of Databases and Information Systems, Ulm University, Germany \\ \{firstname.lastname\}@uni-ulm.de
}

\begin{abstract}
Scalability of information systems has been a research topic for many years and is as relevant as ever with the dramatic increases in digitization of business processes and data. This also applies to processaware information systems, most of which are currently incapable of scaling horizontally, i.e., over multiple servers. This paper presents the design science artifact that resulted from engineering a highly scalable process management system relying on the object-aware process management paradigm. The latter allows for distributed process execution by conceptually encapsulating process logic and data into multiple interacting objects that may be processed concurrently. These objects, in turn, are represented by individual microservices at run-time, which can be hosted transparently across entire server clusters. We present measurement data that evaluates the scalability of the artifact on a compute cluster, demonstrating that the current prototypical implementation of the run-time engine can handle very large numbers of users and process instances concurrently in single-case mechanism experiments with large amounts of simulated user input. Finally, the development of scalable process execution engines will further the continued maturation of the data-centric business process management field.
\end{abstract}

\section{Problem Definition}

For decades, researchers have been examining parallelism, concurrency, and scalability in computer hard- and software. The topic of scalability also became instantly relevant to workflow management systems (WfMS) when they first showed up on the market, as they were explicitly built with large-scale applications in mind [16]. First attempts to create scalable WfMS applied existing scalable architecture principles. The resulting approaches, such as WIDE [7], OSIRIS [17], ADEPTdistribution [5], and Gridflow [6], focused on the system architecture point of view, largely ignoring other aspects, such as role assignments, permissions, and data flow. However, the process models these approaches, especially Gridflow, are meant to support, are typically high-performance production workflows, where these aspects merely play a secondary role [16]. 
Furthermore, with the increasing digitization of business processes and data in recent years, the scalability and speed of process management systems that focus on the execution of human-centric processes has become a relevant topic. This is most noticeable in the widespread availability of commercial cloud-based BPM solutions and even academic implementations [18]. Such cloud-based BPM solutions rely heavily on a highly scalable back end architecture to enable multitenancy without creating individual virtual machines for each customer. Currently, however, no truly hyperscale process execution engine exists that combines a highly scalable back end with a process execution concept that allows more users to work on the same process instance than is possible in traditional, activity-centric process management, where the number of concurrently working users is typically limited by the branches in the process model [4].

This paper presents our in-depth experiences and details of the artifact that shall fill this gap: the PHILharmonicFlows process engine, which is implemented based on the object-aware process management paradigm [13]. Section 3 presents the solution objectives, detailing what we set out to achieve. Before delving into the artifact design and development in Section 4, we establish the necessary fundamentals for object-aware process management in Section 2. The evaluation of the artifact can be found in Section 5. Section 6 gives a brief overview on related work. Finally, Section 7 summarizes the contribution and gives an outlook.

\section{Fundamentals of Object-Aware Process Management}

PHILharmonicFlows, the object-aware process management framework we are using as a test-bed for the concepts presented in this paper, has been under development for many years at Ulm University $[4,13,20,3,14]$. PHILharmonicFlows takes the idea of a data-driven and data-centric process management system, enhancing it with the concept of objects. One such object exists for each business object present in a real-world business process. As can be seen in Fig. 1, an object consists of data, in the form of attributes, and a state-based process model describing the object lifecycle.

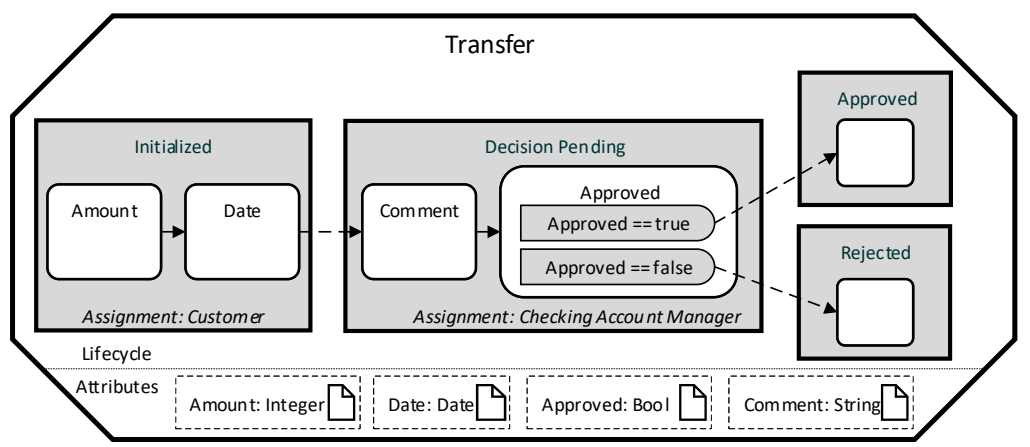

Fig. 1. "Transfer" Object, with Lifecycle Process and Attributes 
The attributes of the Transfer object (cf. Fig. 1) include Amount, Date, Comment, and Approved. The lifecycle process, in turn, describes the different states (Initialized, Decision Pending, Approved, and Rejected), a Transfer object may pass during process execution. In turn, a state contains one or more steps, each referencing exactly one of the object attributes, thereby forcing that attribute to be written at run-time. The steps are connected by transitions, allowing them to be arranged in a sequence. The state of the object changes when all steps in a state are completed. Finally, alternative paths are supported in the form of decision steps, an example of which is the Approved decision step.

As PHILharmonicFlows is data-driven, the lifecycle process for the Transfer object can be understood as follows: The initial state of a Transfer object is Initialized. Once a Customer has entered data for the Amount and Date attributes, the state changes to Decision Pending, which allows an Account Manager to input data for Comment and Approved. Based on the value of Approved, the state of the Transfer object changes to either Approved or Rejected. Obviously, this fine-grained approach to modeling business processes increases complexity compared to the activity-centric paradigm, where the minimum granularity of a user action is one atomic activity or task, instead of an individual data attribute.

Additionally, the object-aware approach allows for automated form generation at run-time. This is facilitated by the lifecycle process of an object, which dictates the attributes to be filled out before the object may switch to the next state, resulting in a personalized and dynamically created form. An example of such a form, derived from the lifecycle process in Fig. 1, is shown in Fig. 2.

Note that a single object and its resulting form only constitutes one part of a PHILharmonicFlows process. To allow for complex executable business processes, many different objects may have to be involved [20]. The entire set of objects and relations present in a PHILharmonicFlows process is denoted as the data model, an example of which can be seen in Fig. 3. In addition to the objects, the data model contains information about the relations existing between them. A relation constitutes a logical association between two objects, e.g., a relation between a Transfer and a Checking Account. The resulting meta information, i.e., the information that the Transfer in question belongs to this specific Checking Account, can be used to

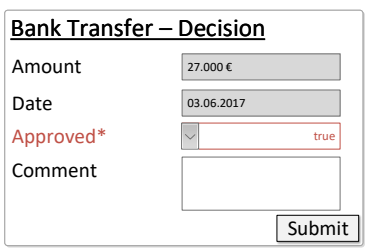

Fig. 2. Example Form

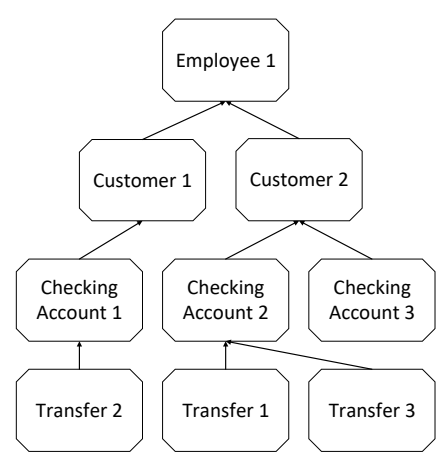

Fig. 3. Data Model coordinate the execution of the two objects.

Finally, complex object coordination, which is indispensable for processes with interacting business objects, is supported as well $[20,19]$. As objects publicly advertise their state information, the current state of an object is utilized as an 
abstraction to coordinate with other objects through a set of constraints, defined in a coordination process. As an example, consider the following constraint: "Only 4 Transfer objects that have a relation to the same Checking Account may be in the Approved state at the same time". A coordination process is always attached to one object, and can coordinate all objects related to that object.

In summary, objects each encapsulate data (i.e., the attributes) and logic (i.e., object behavior) which is based on attribute values (i.e., the lifecycle process). Therefore, objects are largely independent of each other, apart from the relations that exist between them, which are necessary for their orchestration by the coordination processes.

\section{Solution Objectives}

The artifact we present in this work is the distributed object-aware process execution engine PHILharmonicFlows. As stated in the problem definition, there are two main objectives we wish to achieve with this artifact: develop a highly scalable process management system, which uses an underlying process management concept that allows for a multitude of users to work concurrently on the same process instance (i.e., Objective 1) and is engineered in a way so that it scales out well when more hardware resources are added (i.e., Objective 2).

Objective 1 can be achieved by breaking with the traditional activity-centric process management approach, i.e., employing an approach such as artifactcentric [8], case handling [1], or object-aware process management [14]. Due to the fact that in such approaches the tasks users have to perform are not shoehorned into atomic activities, such as forms, which only one person at a time can view and edit, they are intrinsically more scalable from a user point of view as more users may execute parts of process instances concurrently [4].

Objective 2, engineering the process engine in a way such that it becomes highly scalable in regards to the hardware it runs on, requires a more precise explanation. First, it is necessary to clarify that the goal is not to create a system that scales up well, i.e. vertically with a more powerful processor, but scales out well horizontally with more computers added to a data center or computing cluster, as this is generally more desirable in cloud scenarios. In particular, we aim at achieving ideal linear speedup, i.e., increasing the amount of available processing power by factor $p$ should speed up process execution by $p$ [12].

Finally, while one can design an activity-centric process management engine in a way that allows it to scale well on the hardware side, the benefits of combining the necessary software architecture aspects with the conceptual aspects of objectaware process management are what make the artifact original.

\section{Design and Development}

The PHILharmonicFlows process engine has undergone many development cycles, starting with an extensive research phase concerning the conceptual foun- 
dations (cf. Section 2). The most important architectural iteration concerned the scalability aspects described in this paper.

\subsection{Architectural Challenges}

Initially, we had opted for a relational database to hold the process execution state, as it is common in many other process engines. However, during the testing phase of the development iteration, we noticed that the prototype was plagued by severe scalability issues when confronting it with large numbers of concurrent users and objects. Note that, as opposed to more traditional (i.e., activity-centric) process management technology, object-aware process management needs to react to very fine-grained user actions. Due to the nature of the lifecycle processes and the dynamically generated forms, the engine has to react to each data element input by users at run-time. In consequence, there is a much higher frequency at which small workloads have to be completed by the process engine. Note that this is the price one has to pay for the increase in flexibility compared to activity-centric systems, which use predefined forms and allow for far fewer possible execution variants.

As mentioned, a relational database is not ideally suited as the backbone for such a data-centric and data-driven system, as each of the actions a user completes on one object may have effects on multiple other objects, each represented by rows in database tables. As a consequence, a large number of rows (for various attributes, objects, and relations) have to be loaded into memory to determine the effects of a user action. In particular, if actions lead to large amounts of small changes to other objects, time is wasted on locking/unlocking rows and tables, slowing the system down considerably. Furthermore, the necessary communication between the individual objects is not predictable up front, as it depends on (1) the coordination process, (2) the structure of the data model, i.e., the relations that exist between the objects in question, and (3) the current state of each object. As these factors may change at any time during process execution, common techniques (e.g. relying on query optimization) are not ideal for our use case. In consequence, at least conceptually, object-aware process management has an unpredictable $n-m-n$ communication pattern, a very simple depiction of which can be seen in Fig. 4. After carefully examining this unpredictable pattern we opted for a distributed approach to persisting the state of objects.

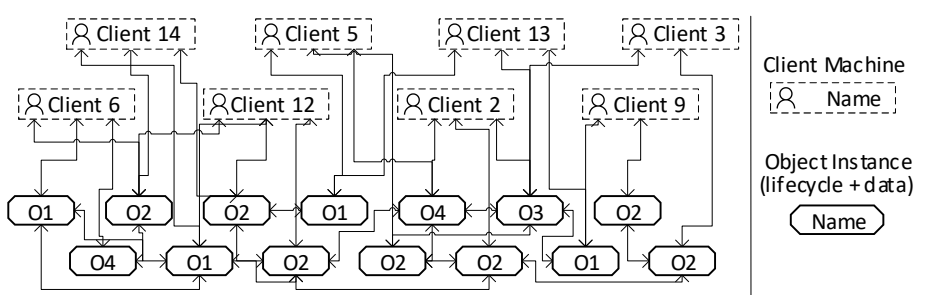

Fig. 4. Object Communication Pattern (Simplified) 


\subsection{Design Methodology}

After experimenting with highly distributed and lightweight document databases, such as RethinkDB and Couchbase, and not achieving satisfying performance results, we decided to not only distribute the persistence layer, but also the computation. To facilitate this, we applied actor model theory. The actor model is a well established theoretical foundation for concurrency in parallel systems in which the computation and persistence layer are split into largely independent primitives called actors [2]. Furthermore, it is supported by a number of frameworks for highly scalable applications and supports communication patterns such as the one present in object-aware process management (cf. Fig. 4).

Background on Actor Model Theory The following gives an overview on the theory behind the actor model. In essence, an actor consists of a message queue and a store for arbitrary data. The actor can receive messages and handle them using the data from its store or by sending messages to other actors. An actor, however, may only complete exactly one task at a time, i.e., an actor currently servicing a request from its message queue may only work on answering that one message, whereas all others are ignored until the current message is removed from the queue. An actor system usually consists of a very large number of actors of different types, which each type having different functionality which can be completed by any instances of that type.

An example of the communication between a set of actors of different types, A, $\mathrm{B}$, and $\mathrm{C}$, can be seen in Fig. 5. Note that actor A receives a request from an external source, depicted by the message in its message queue. As each actor represents a small unit of functionality and data, an actor of type A might not have all the information or functionality to service the request, which necessitates communication with the other actors, e.g., B and C. However, the part of the request redirected at actor $\mathrm{B}$ triggers communication between Actor $\mathrm{B}$ and actor C. Due to the single conceptual "thread", as well as the forced message queuing, most concurrency problems concerning persistence and computation, such as race conditions and dirty read/writes, cannot occur in an actor system.

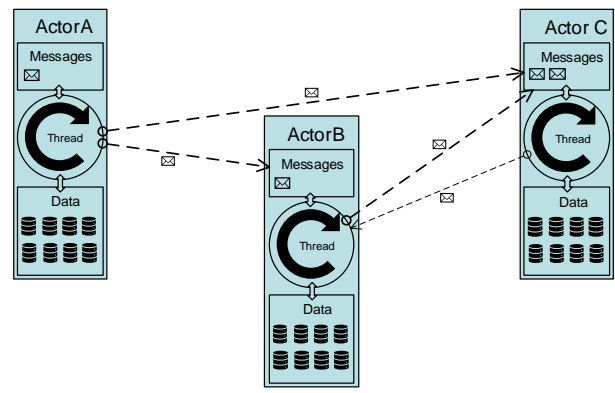

Fig. 5. Actor Communication 
Applying the Actor Model While the actor model may not be fitting for systems with few, yet long-running, tasks, it is well suited to handle the challenges posed by a multitude of communicating and interacting objects present in an object-aware data model. While the actor model itself guarantees a highly scalable distributed system with few concurrency problems, the main challenge is to actually apply it to an existing concept or software system. In the context of our work, this means finding the correct mapping of the various conceptual elements of object-aware process management to individual actors. To this end, we elect to treat each object in a data model as an actor, which allows us to keep the conceptual elements contained in the object, i.e., the lifecycle process on the computation side, and the the attributes on the persistence side, together as one unit. Note that this ensures that updates to individual objects, such as changes to attribute values (and the resulting updates to the lifecycle processes), can be handled independently from unrelated objects.

Note that the coordination processes (cf. Section 2) coordinate the various objects based on (1) their current state as well as (2) their relations to other objects. To enable (1), objects that advance to a different state after one of their attribute values is changed must inform their coordination process of the respective state change. We facilitate this by leveraging the actor message pattern described in Section 4.2. To enable (2), we encapsulate the relations themselves as actors. This allows the actor representing a new relation to send a message informing the coordination processes of its creation. Finally, to ensure that the coordination processes handle such messages in the same way as objects, i.e., ordered and race condition free, we redesigned them to be actors as well.

To be precise, all high-level conceptual elements present in object-aware processes, i.e., objects, relations, coordination processes, and the data model, are represented as actors in the PHILharmonicFlows engine. As the actor model allows for independent execution of logic, using only wellregulated message exchanges at certain points, actors can be hosted as individual microservices in computation environments. This results in a five layer concept for the process management engine, a sketch of which is shown in Fig. 6. As Fig. 6 implies, all high-level conceptual elements of object-

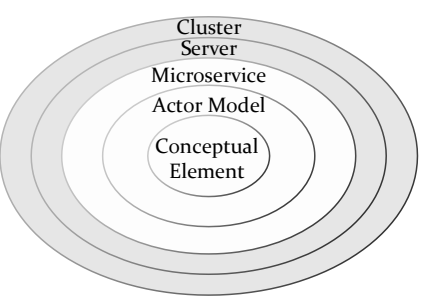

Fig. 6. Encapsulation Diagram of Conceptual Elements, Actors, and Microservices aware process management can be interpreted as actors, allowing them to be hosted in microservices on a server that can be part of a cluster. Clearly, Fig. 6 abstracts from the fact that multiple instances of any conceptual element may exist, resulting in one microservice per instance of the conceptual element existing at run-time.

Note that we chose to keep the more fine-grained conceptual elements, such as attributes, permissions, roles, and lifecycle states and steps as state information inside the actors representing the objects. Taking the checking of an attribute 
permission as an example, as shown in Fig. 7, it becomes evident why having the permissions and attributes as independent actors would make little sense.

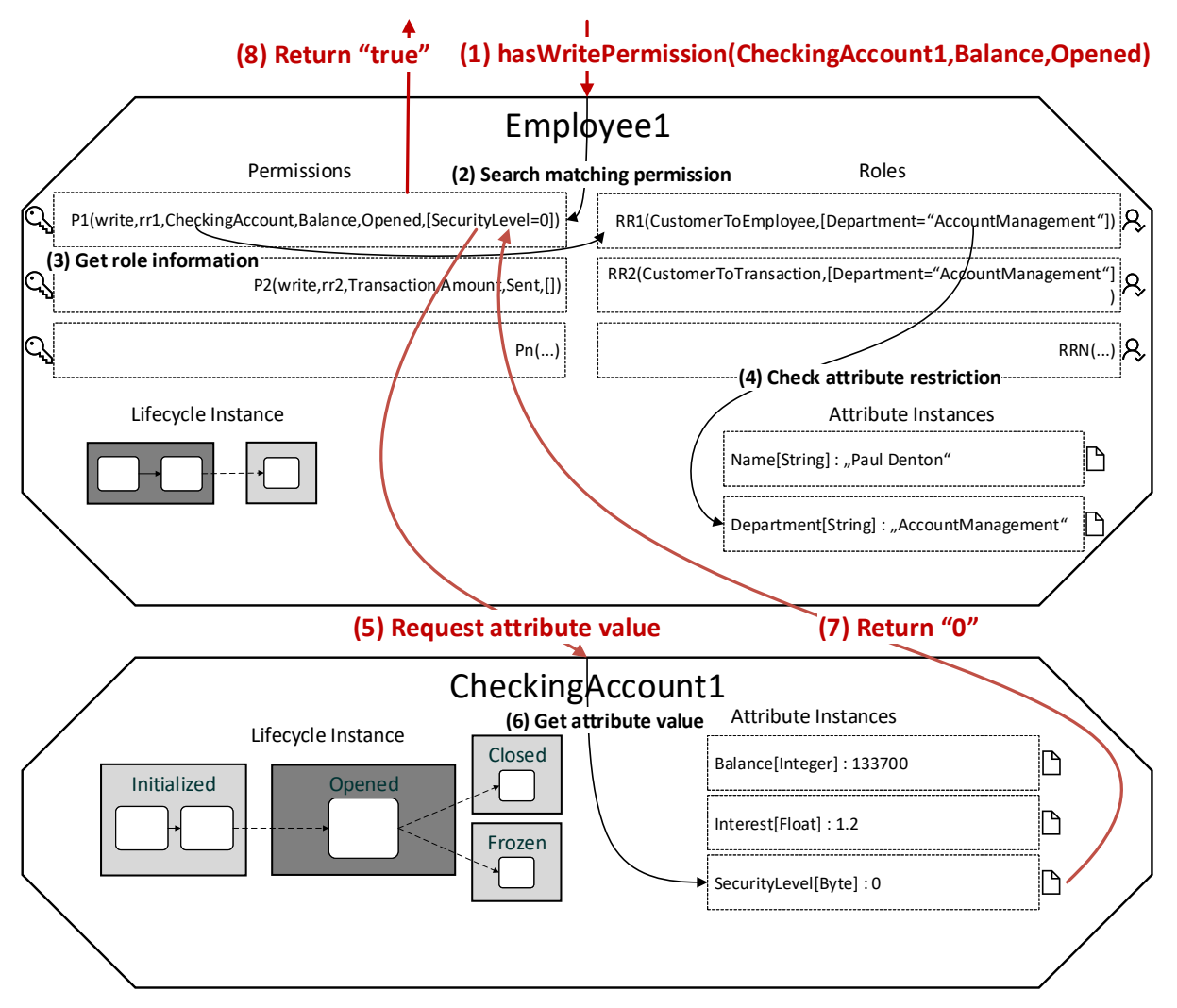

Fig. 7. Permission Request

In the example, two objects, Employee1 and CheckingAccount1, each represented by an actor at run-time, are shown. Note that only markings (1), (5), (7), and (8) constitute messages being passed between actors, all other markings are just operations that are completed using data local to the respective actor, the details of which are not relevant at this point, but can be found in previous work [3]. After the initial request (1) arrives in the message queue of Employee1, it has to complete a number of operations. Furthermore, this specific request causes the Employee1 actor to communicate (5) with the CheckingAccount1 actor. In particular, granting the permission depends on the value of the SecurityLevel attribute, which is only present in the actor for CheckingAccount1. Finally, after actor CheckingAccount1 returns the value of the attribute (7), actor Employee1 can return the result of the request (8). 
Obviously, as the two actors for Employee1 and CheckingAccount1 may be located on different servers in the computing cluster, a request like this can introduce some communication overhead. However, as our goal is not to create an artifact that can resolve a single request to an object-aware process engine as fast as possible, but instead scales out well, this overhead is negligible, as shown by our measurement results in Section 5. A more fine-grained decomposition, e.g., putting every permission, attribute, and step of the lifecycle process into individual actors, would render no additional benefit as the information they represent is often only needed by the object they belong to.

In summary, we chose the actor model as a basis for the PHILharmonicFlows engine as it fits well to the already established conceptual elements of object-aware process management. Furthermore, as each actor maintains its own which solely this actor may access, we chose the granularity of decomposition of conceptual elements to actors in a way such that requests to an actor, e.g., an object, can be serviced without too much communication overhead. Moreover, having all data of an object located in the corresponding actor prevents common concurrency problems, such as dirty reads on the attributes, as the data may only be accessed by one incoming request to the actor at the same time. Finally, the architecture, which is based on the actor model allows us to host each actor in its own microservice. As microservices enable high degrees of horizontal scalability, any computational logic that can be partitioned into individual actors can be scaled across multiple servers well. This means that scaling out the PHILharmonicFlows engine horizontally is possible on the fly, which makes it ideal for scalable business process execution in cloud scenarios.

Architecture Several actor model based microservice frameworks exist, notable ones include $\mathrm{Akka}^{1}$ (Java), $\mathrm{CAF}^{2}(\mathrm{C}++)$, and Orleans ${ }^{3}$ (.NET). As the preexisting code base of PHILharmonicFlows was written in .NET, we utilize a modified version of Orleans, which is part of the open source Service Fabric Reliable Actors $\mathrm{SDK}^{4}$, for our prototype. Service Fabric allows us to run actor-based applications on development machines, on-premise research computing clusters, and in the cloud. This is enabled by a transparent placement of instantiated actors across all available servers. A diagram of the entire architecture of our engine is shown in Fig. 8. The "Actor Services" and "Framework Services" are hosted across all servers in the cluster, ensuring that they are all fully capable of instantiating actor microservices, such as those shown above "Instantiated Microservices". All depicted elements are implemented and working to the specifications of objectaware process management. Finally, note that the engine is still only considered a prototype for our artifact, as we have not yet conducted technical action research with real-world clients.

\footnotetext{
${ }^{1}$ https://github.com/akka

2 https://github.com/actor-framework

${ }^{3}$ https://github.com/dotnet/orleans

${ }^{4}$ https://github.com/Azure/service-fabric-services-and-actors-dotnet
} 


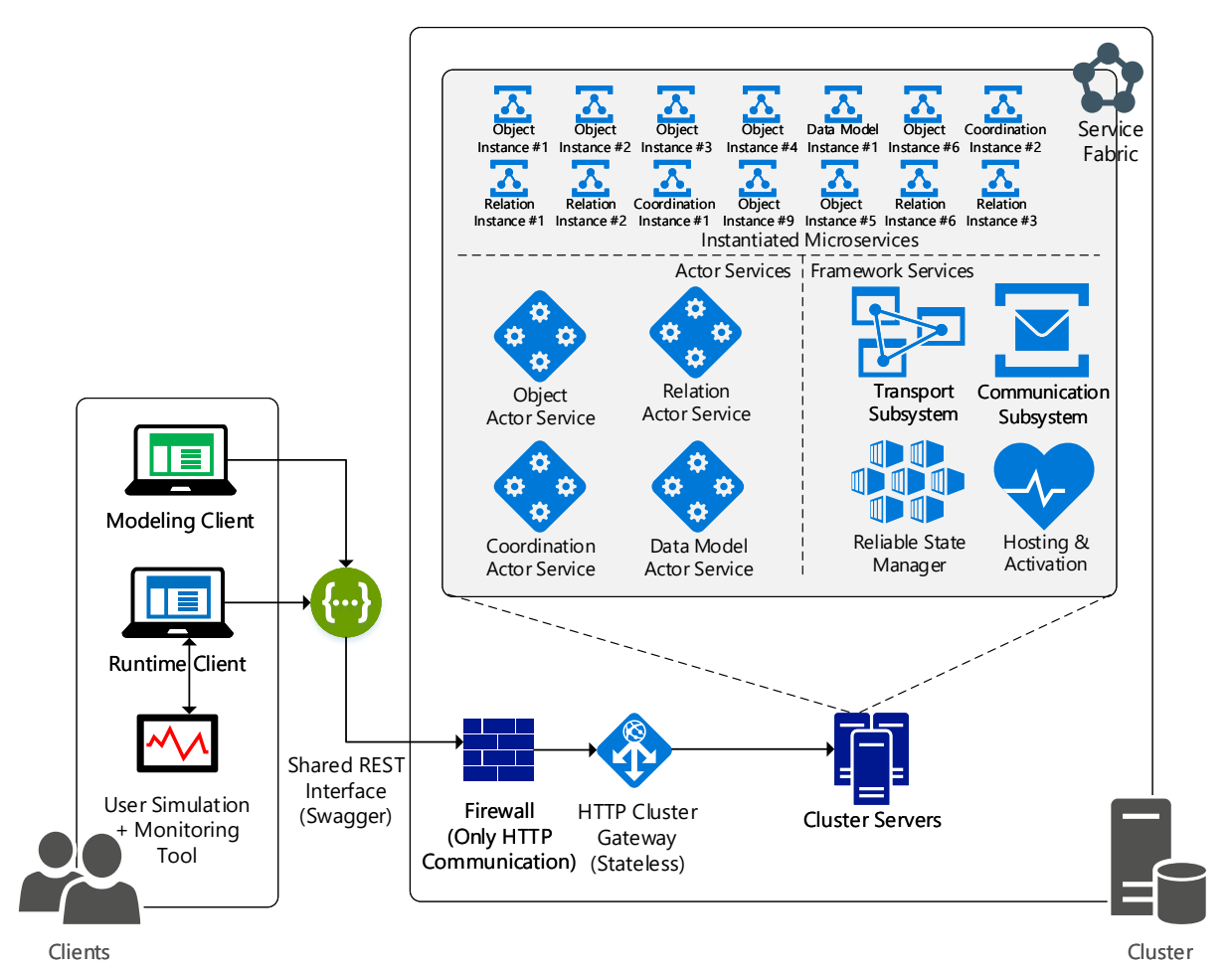

Fig. 8. Architecture Overview

\section{Evaluation and Demonstration}

As other aspects of the PHILharmonicFlows process management system are still under development, both conceptually and from an implementation perspective, we have not completed technical action research to verify the solution objectives. Instead, we are currently conducing single-case mechanism experiments to evaluate the capabilities of the developed architecture, the results of which we present in this section. Before, however, we discuss conceptual performance limitations of object-aware processes as well as the measurement methodology.

\subsection{Conceptual Performance Limitations}

As each object in an object-aware data model can be interacted with individually, there are hardly any bottlenecks to be expected for the execution of the object lifecycle processes. However, there is the issue of coordinating the execution of inter-related objects that needs particular consideration. In general, coordination processes are informed by the objects when their states change due to the completion of steps in their lifecycle processes. How often this happens 
depends on the lifecycle process model of the object in question. In particular, as these are merely fire-and-forget messages to the coordination process, they do not actually impact the performance of the object.

Obviously, coordination processes with constraints, such as the example given in Section 2, are necessary to model real-world processes. However, they do not constitute a scalability issue in the computing sense, as it is often necessary to wait for other parts of a process to complete in any process management paradigm. To enable such coordination constraints, it has to be clear which objects are linked to each other, i.e. have relations between them [19]. The structure of all objects and the relations between them constitute the data model of an object-aware process. As all relations are unidirectional and acyclic, the data model forms a tree-like directed acyclic graph structure, as shown in Fig. 3.

The central performance limitation of the artifact stems from the fact that linking a new object to the existing data model causes a recursive update all the way to the root object. This is necessary since any objects with coordination processes on higher levels of the data model have to be informed of new objects being attached on lower levels in order to coordinate them properly. To ensure that this operation is completed in an ordered fashion, it is completed by the actor representing the data model at run-time. As actors may only complete one action at a time, the linking of objects constitutes a performance bottleneck. However, objects can only create relations to other objects as specified at design time in the process model. Thus, the number of relations creatable at run-time is directly limited by the business process itself. In summary, as in most cases objects are only related to one or two other objects directly (others transitively), only few relation creation operations are conducted for each object at run-time.

\subsection{Experiment Scenario and Tooling}

As our engine is an actor system running on Service Fabric (cf. Section 4.2), it can be deployed to a cluster with an arbitrary number of servers. For our singlecase mechanism experiments, we employ an on-premise cluster with eight servers and a total of 64 logical processors (32 cores @ 2.4Ghz + hyper-threading). The data model used for the experiments is the Banking scenario presented in Section 2 , an example of a typical object-aware process model.

Obviously, an object must be instantiated in a microservice before it can be linked to other objects or be manipulated through attribute value changes. However, the order in which the attributes of an object are set, or whether they are set before or after linking the object to others, has no influence on outcome after all actions are completed. This is due to the fact that object-aware execution is data-driven, which allows us to design the experiment as follows: (1) we instantiate all objects required for the scenario a predefined number of times, (2) we link the objects together using a predefined schema, and, finally, (3) we supply predefined attribute values to each object we instantiated, thereby executing their lifecycle processes. Our tooling simulates user interaction directly through the run-time client via REST, using the architecture shown in Fig. 8. 
We can measure times for all three action types, i.e., instantiation (1), linking (2), and execution (3), as well as the total time for a run. This allows us to discuss the three distinct actions and to observe the impact of changing various parameters on the time they take in relation to the total time.

\subsection{Statistical Measurement Methodology}

The measurements follow the guidelines for measuring the performance of parallel computing systems, as defined in [12]. As a parallel computing system can render vastly different numbers for the same experiment scenario when run multiple times, it is hard to measure exact execution times.

Instead of simply measuring the mean or median execution times over a predefined arbitrary number of runs, we use the statistically exact method presented in [12] to determine the number of runs necessary to ensure high confidence in an adequately small confidence interval. As we are dealing with non-normally distributed data, the number of runs has to be determined dynamically i.e., there is no analytical method for predetermining it. As it can take several hundred minute long runs to get a narrow confidence interval, this exact calculation becomes necessary to not waste hours trying to find an adequate number of runs.

To this end, we run Algorithm 1 after every individual experiment run to ensure that after $n$ runs the confidence interval is tight enough and the confidence in the interval is high enough. The inputs are the already completed experiment runs (runs), the maximum width of the confidence interval as a percentage of the confidence interval average $\left(\%_{C I}\right)$, and the minimum confidence in the confidence interval $\left((1-\alpha)_{\text {min }}\right)$. The algorithm returns interval $\left[\right.$ time $_{\text {lRank }}$, time $\left._{u \text { Rank }}\right]$, i.e., the total time of the runs at the lower and upper bounds of the confidence interval, as well as $(1-\alpha)$, i.e., the exact confidence level of the returned interval. Note that Algorithm 1 uses the statistical method described in [15], Annex A, to determine which of the already measured runs constitute the bounds of the confidence interval with the required confidence $(1-\alpha)_{\min }$.

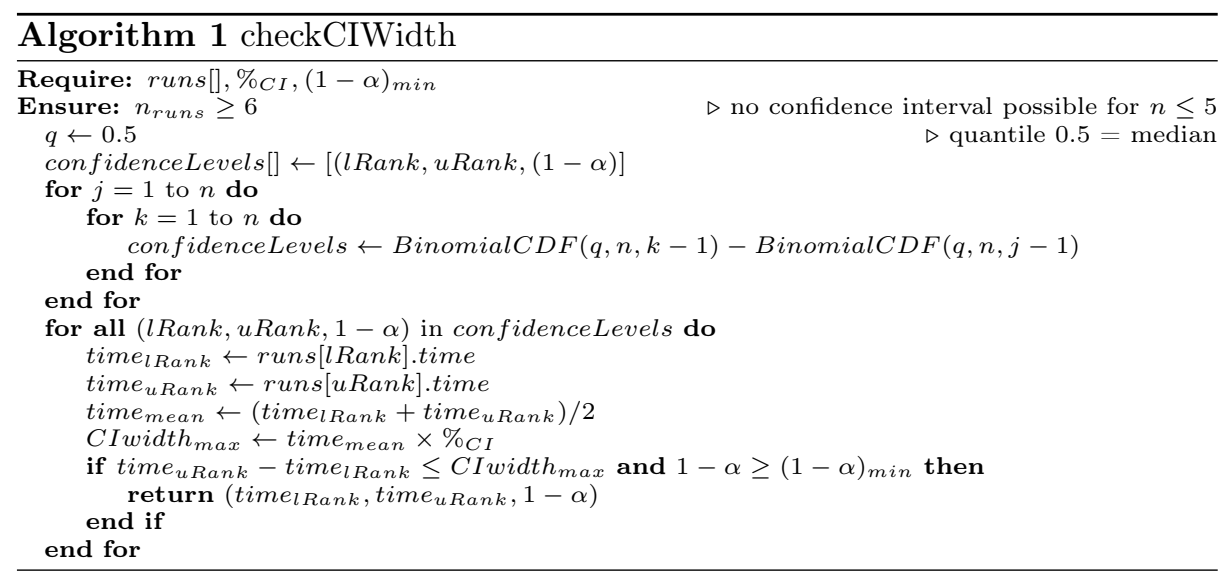




\subsection{Results}

We evaluated how well the artifact achieves the solution objectives (cf. Section 3) based on the measurement results from the single-case mechanism experiments presented in this section. The objective of the experiments was (1) to determine the limits of how many concurrent objects can be processes on the given eight server hardware setup, (2) find bottlenecks by simulating mass user interactions and (3) measure the impact of object lifecycle complexity on execution times.

To this end, we conducted the following series of single-case mechanism experiments with an example process model and simulated user input to the current PHILharmonicFlows engine prototype. While this is not technical action research in the real world, as explained in Section 5.2, the engine is interacted with exactly as if thousands of real users were executing the individual objects concurrently. We utilize these results to demonstrate the viability of a highly scalable actor model based microservice architecture for data-centric process management systems in general.

Each results table row represents an experiment series with a specific configuration and the following in- and outputs:

\begin{tabular}{|c|c|l|}
\cline { 2 - 3 } \multicolumn{1}{c|}{ Header } & Description \\
\hline$n_{D}$ & number of data models created \\
\hline$n_{O}$ & number of created objects across all data models \\
\hline$n_{R}$ & number of created relations across all data models \\
\hline$C I t_{\text {inst }}$ & confidence interval for total instantiation time \\
\hline & $C I t_{\text {link }}$ & confidence interval for total linking time \\
\hline$C I t_{\text {exec }}$ & confidence interval for total lifecycle execution time \\
\hline$C I t_{\text {total }}$ & confidence interval for total run time of experiment \\
\hline $1-\alpha$ & exact confidence in the $C I t_{t o t a l}$ confidence interval \\
\hline$n$ & number of runs completed to achieve $1-\alpha$ confidence in $C I t_{\text {total }}$ \\
\hline \multirow{2}{*}{} & & nelation function, A:B:100 indicates 100 relations between objects \\
\hline
\end{tabular}

All confidence intervals in all result tables are required to have a maximum width of $5 \%$ in respect to the median measured time, i.e., $\%_{C I}=0.05$. Further, the confidence in each interval must be at least $95 \%$, i.e., $1-\alpha_{\min } \geq 0.95$. As this means that a measurement with a suspected median time of $1000 \mathrm{~ms}$ is required to have a confidence interval of $[975 \mathrm{~ms}, 1025 \mathrm{~ms}]^{5}$, and the confidence that the real median time lies in this interval must be at least $95 \%$. Finally, all time measurements are specified in the mm:ss.ms format. Table 1 shows the measurement results for a scenario in which we instantiate, link, and execute varying numbers of Checking Accounts (CA) and Transfers (TR).

\footnotetext{
${ }^{5} 50 \mathrm{~ms}$ width, $5 \%$ of $1000 \mathrm{~ms}$
} 
Table 1. Simple Data Model: Checking Accounts (CA) and Transfers (TR)

\begin{tabular}{|c|c|c|c|c|c|c|c|c|c|c|}
\hline$n_{D}$ & $O$ & $R$ & $n_{O}$ & $n_{R}$ & $C I t_{\text {inst }}$ & $C I t_{l i n k}$ & $C I t_{\text {exec }}$ & $C I t_{\text {total }}$ & $1-\alpha$ & $n$ \\
\hline 1 & $\begin{array}{l}\text { CA:100, } \\
\text { TR:1000 }\end{array}$ & TR:CA:1000 & 1100 & 1000 & $\begin{array}{c}\text { [00:00.960, } \\
\text { 00:01.003] }\end{array}$ & $\begin{array}{c}{[00: 03.002,} \\
00: 03.190]\end{array}$ & $\begin{array}{l}{[00: 00.422,} \\
00: 00.603]\end{array}$ & $\begin{array}{c}{[00: 04.499} \\
00: 04.969]\end{array}$ & 96.49 & 32 \\
\hline 1 & $\begin{array}{l}\text { CA:500, } \\
\text { TR:5000 }\end{array}$ & TR:CA:5000 & 5500 & 5000 & $\begin{array}{c}{[00: 04.452,} \\
00: 04.540]\end{array}$ & $\begin{array}{c}{[00: 14.037} \\
00: 14.984]\end{array}$ & $\begin{array}{l}{[00: 01.898,} \\
00: 03.622]\end{array}$ & $\begin{array}{c}{[00: 21.075} \\
00: 23.272]\end{array}$ & 95.67 & 24 \\
\hline 1 & $\begin{array}{l}\text { CA:1000, } \\
\text { TR:10000 }\end{array}$ & TR:CA:10000 & 11000 & 10000 & $\begin{array}{c}{[00: 08.657} \\
00: 08.993]\end{array}$ & $\begin{array}{c}{[00: 29.127} \\
00: 33.835]\end{array}$ & $\begin{array}{l}{[00: 04.499,} \\
00: 06.333]\end{array}$ & $\begin{array}{c}{[00: 42.898} \\
00: 46.550]\end{array}$ & 96.14 & 11 \\
\hline 2 & $\begin{array}{l}\text { CA:500, } \\
\text { TR:5000 }\end{array}$ & TR:CA:5000 & 11000 & 10000 & $\begin{array}{c}\text { [00:04.723, } \\
\text { 00:04.979] }\end{array}$ & $\begin{array}{c}{[00: 16.713,} \\
00: 18.122]\end{array}$ & $\begin{array}{l}{[00: 02.810,} \\
00: 04.482]\end{array}$ & $\begin{array}{c}{[00: 24.648,} \\
00: 27.076]\end{array}$ & 95.67 & 25 \\
\hline 4 & $\begin{array}{l}\text { CA: } 250, \\
\text { TR:2500 }\end{array}$ & TR:CA:2500 & 11000 & 10000 & $\begin{array}{c}{[00: 02.943} \\
00: 03.307]\end{array}$ & $\begin{array}{c}{[00: 11.161} \\
00: 12.908]\end{array}$ & $\begin{array}{l}{[00: 02.707,} \\
00: 04.004]\end{array}$ & $\begin{array}{c}{[00: 17.742,} \\
00: 19.554]\end{array}$ & 95.86 & 20 \\
\hline 8 & $\begin{array}{l}\text { CA: } 125, \\
\text { TR:1250 }\end{array}$ & TR:CA:1250 & 11000 & 10000 & $\begin{array}{c}{[00: 04.130} \\
00: 04.516]\end{array}$ & $\begin{array}{c}{[00: 10.109,} \\
00: 10.670]\end{array}$ & $\begin{array}{l}{[00: 03.239,} \\
00: 05.202]\end{array}$ & $\begin{array}{c}{[00: 18.493,} \\
00: 20.439]\end{array}$ & 95.3 & 37 \\
\hline
\end{tabular}

A few things are noteworthy here. First, regarding the first four series, in which all objects and relations were part of a single data model, one can observe almost ideal linear scaling of the various median times with the respective workloads, as evidenced by Fig 9. This demonstrates the capability of the microservices on the cluster to handle increasing concurrent workloads extremely well. Any below linear curve would indicate the system becoming congested.

Second, note the super-linear scaling of $C I t_{\text {link }}$ in the four series with 10.000 relations spread over an increasing number of data models (cf. Fig. 10). This indicates a conceptual bottleneck due to the fact that the linking operations must be ordered to ensure correct recursive updating of the entire data model, as explained in Section 5.1. To this end, each linking operation is queued and executed by the actor representing the data model in question, reducing concurrency.

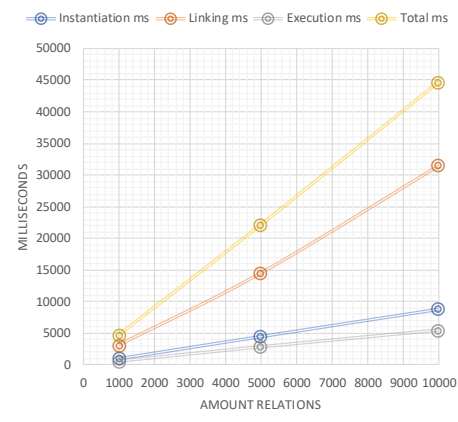

Fig. 9. Single Data Model

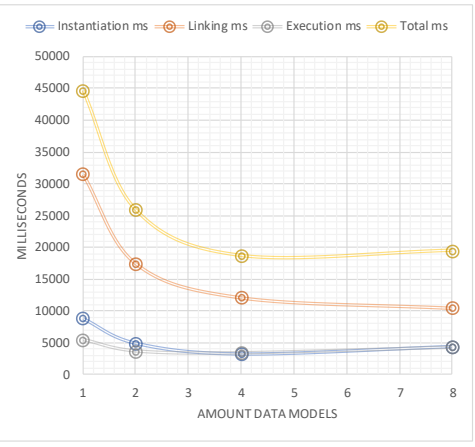

Fig. 10. Multiple Data Models 
Table 2. Complex Data Model: Employees (EM), Customers (CU), Checking Accounts (CA), and Transfers (TR)

\begin{tabular}{|c|c|c|c|c|c|c|c|c|c|c|}
\hline$n_{D}$ & $O$ & $R$ & $n_{O}$ & $n_{R}$ & $C I t_{i n s t}$ & $C I t_{l i n k}$ & $C I t_{\text {exec }}$ & $C I t_{\text {total }}$ & $1-\alpha$ & $n$ \\
\hline 1 & $\begin{array}{c}\text { EM:1, } \\
\text { CU:10, } \\
\text { CA:100, } \\
\text { TR:1000 }\end{array}$ & $\begin{array}{l}\text { CU:EM:10, } \\
\text { CA:CU:100, } \\
\text { TR:CA:1000, }\end{array}$ & 1111 & 1110 & $\begin{array}{c}\text { [00:00.926, } \\
00: 00.993]\end{array}$ & $\begin{array}{c}{[00: 05.424,} \\
00: 06.160]\end{array}$ & $\begin{array}{c}{[00: 00.418} \\
00: 00.482]\end{array}$ & $\begin{array}{c}{[00: 06.892,} \\
00: 07.573]\end{array}$ & 96.48 & 14 \\
\hline 1 & $\begin{array}{c}\text { EM:5, } \\
\text { CU:50, } \\
\text { CA:500, } \\
\text { TR:5000 }\end{array}$ & $\begin{array}{c}\text { CU:EM:50, } \\
\text { CA:CU:500, } \\
\text { TR:CA:5000, }\end{array}$ & 5555 & 5550 & $\begin{array}{c}{[00: 04.518} \\
00: 04.754]\end{array}$ & $\begin{array}{c}{[00: 27.701} \\
00: 30.809]\end{array}$ & $\begin{array}{c}{[00: 03.800} \\
00: 05.538]\end{array}$ & $\begin{array}{c}{[00: 37.184} \\
00: 40.179]\end{array}$ & 96.09 & 9 \\
\hline 1 & $\begin{array}{c}\text { EM:10, } \\
\text { CU:100, } \\
\text { CA:1000, } \\
\text { TR:10000 }\end{array}$ & $\begin{array}{l}\text { CU:EM:100, } \\
\text { CA:CU:1000, } \\
\text { TR:CA:10000, }\end{array}$ & 11110 & 11100 & $\begin{array}{c}{[00: 09.110} \\
00: 09.380]\end{array}$ & $\begin{array}{c}{[00: 57.553,} \\
01: 02.829]\end{array}$ & $\begin{array}{c}{[00: 06.504,} \\
00: 08.429]\end{array}$ & $\begin{array}{c}{[01: 12.990} \\
01: 19.123]\end{array}$ & 96.14 & 12 \\
\hline
\end{tabular}

Table 2 shows the experiment results when creating a significantly more complex data model, with all objects and relations shown in Fig. 3. The objects involved are Checking Account (CA), Employee (EM), Transfer (TR), and Customer (CU). However, we adjusted the total amount of objects created to be almost identical to the experiment series shown in the first three rows of Table 1. Note the large increase in the $C I t_{\text {total }}$ interval when comparing Table 2 with Table 1. However, the increase clearly stems from the higher effort when linking new objects into the more complex data model, as evidenced by the higher $C I t_{\text {link }}$ values, as the average $C I t_{\text {inst }}$ and $C I t_{\text {exec }}$ values are similar.

Second, consider the wide $C I t_{\text {exec }}$ intervals in comparison to the very tight $C I t_{\text {inst }}$ intervals (only the $C I t_{\text {total }}$ interval width is fixed at $5 \%$ ). This can be explained with the fact that, while both instantiation and execution are tied to the complexity of the respective lifecycle process, instantiation is done by the actor service, while execution is done by the thousands of object actors created during instantiation and spread across the cluster. As we only have four actor services active during the experiment series shown in Table 2, the actor framework will distribute them to four distinct servers for each experiment run. As every server is equal, this leads to almost identical measured times across all experiments. However, as the underlying actor framework does not have knowledge of the varying complexity of the lifecycles processes in the different objects, the thousands of objects will also be distributed at random across the eight servers. In consequence, as some objects take longer to execute their lifecycles than others, servers might be unequally tasked in this scenario, leading to comparatively wide confidence intervals.

Consider the following as a very simplified and extreme example of this effect: objects of type A may take one second to execute their lifecycle process, whereas objects of type B may take ten seconds to execute their lifecycle process. If there 
are two servers and ten objects of each type, in one experiment run both servers might be tasked with executing five objects of type A and five objects of type B, which would take 55 seconds in total. Another run might see all objects of type A executed by one server, taking ten seconds, and all objects of type B executed by the other server, taking 100 seconds.

To further examine these differences in execution times, we instantiated and executed each object type used in Table 210.000 times. The results of this experiment can be seen in Table 3. As there are no relations in this experiment, we instead included the number of attributes, steps, and states the objects possess, as they give an indication as to how complex their lifecycle processes are. The results show the clear correlations between lifecycle complexity and instantiation/execution time.

Table 3. Comparison of Object Performance: Employees (EM), Customers (CU), Checking Accounts (CA), and Transfers (TR)

\begin{tabular}{|c|c|c|c|c|c|c|c|c|c|}
\hline$n_{D}$ & $O$ & Attributes & Steps & States & $C I t_{\text {inst }}$ & $C I t_{\text {exec }}$ & $C I t_{\text {total }}$ & $1-\alpha$ & $n$ \\
\hline 1 & CA: 10000 & 1 & 3 & 3 & $\begin{array}{c}{[00: 06.537} \\
00: 06.757]\end{array}$ & $\begin{array}{c}{[00: 04.076} \\
00: 04.753]\end{array}$ & $\begin{array}{c}{[00: 10.731} \\
00: 11.529]\end{array}$ & 95.1 & 16 \\
\hline 1 & EM:10000 & 2 & 4 & 3 & $\begin{array}{c}{[00: 08.158} \\
00: 08.726]\end{array}$ & $\begin{array}{c}{[00: 04.415} \\
00: 05.857]\end{array}$ & $\begin{array}{c}{[00: 12.804,} \\
00: 14.118]\end{array}$ & 96.87 & 6 \\
\hline 1 & TR:10000 & 4 & 6 & 4 & $\begin{array}{c}{[00: 08.350} \\
00: 08.723]\end{array}$ & $\begin{array}{c}{[00: 06.095} \\
00: 07.521]\end{array}$ & $\begin{array}{c}{[00: 14.499,} \\
00: 15.929]\end{array}$ & 95.72 & 30 \\
\hline 1 & $\mathrm{CU}: 10000$ & 8 & 11 & 4 & $\begin{array}{c}{[00: 11.974} \\
00: 12.358]\end{array}$ & $\begin{array}{c}{[00: 11.222} \\
00: 13.784]\end{array}$ & $\begin{array}{c}{[00: 23.576,} \\
00: 25.979]\end{array}$ & 95.64 & 41 \\
\hline
\end{tabular}

\section{Related Work}

There is a large amount of literature concerning the scalability of software systems, little of which is concerned with process management systems. While the works mentioned in the introduction, WIDE [7], OSIRIS [17], ADEPTdistribution [5], and Gridflow [6] are fairly dated and do not take modern architectures into consideration, there is some newer research that does.

[9] and [11] present architectures for distributing business process workloads between a client-side engine and a cloud-based engine, taking into consideration that users might not want to store their business data in the cloud. Thus, the approaches suggest to primarily run compute-intensive workloads on the cloudbased engine and transfer business data only when necessary. [9] further presents a method for decomposing the process model into two complementary process models, one for the client engine, and one for the cloud engine.

[10] introduces a resource controller for cloud environments that monitors process execution and can predict how compute intensive future workloads will be 
depending on previous executions. This data is used for auto-scaling the virtual machines the process engine and external systems are running on.

[18] gives an in-depth overview of the state of the art concerning cloud business process architecture, revealing that most current approaches use virtual machine scaling, simply replicating process engines to achieve scalability.

\section{Summary}

In this work, we demonstrated the viability of creating a hyperscale process management system using a data-centric approach to process management on the conceptual side as well as actor model theory and microservices on the architecture side. Thus, we maximize the possible concurrent actions conducted by process participants at any given time during process execution, without simply relying on virtual machine scaling as most of the related work does. Our measurements show that the PHILharmonicFlows engine scales linearly with the number of objects created and executed (i.e., user-generated workload) in a single process instance. Furthermore, the measurements give insights into what the engine is capable of, with even this first prototype with no "professional" optimization executing the lifecycle processes of 11.000 objects in around five seconds. This is even more impressive considering the fact that these are full executions of the respective lifecycle processes. As the example objects have an average of 4-5 attributes and steps this equates to around 50.000 user interactions the engine is handling in the aforementioned five second time frame.

While the data from our single-case mechanism experiments shows that datacentric process engines have the potential to scale extremely well and to handle many users working on a single process instance at the same time, we identified a conceptual bottleneck in the linking of newly created objects to the existing data model. As this has to be handled centrally by the data model, it cannot be done concurrently. However, in a more real-world scenario with more running data models and less objects per data model, this is not much of an issue. This is especially true when considering that in general the ratio of object instantiations and linking to actual execution actions, such as form inputs by users, will be low.

In future work, we will work on optimizing the engine further and subjecting it to more real-world usage when conducing technical action research. Another important task is comparing the performance of PHILharmonicFlows to activitycentric process management systems, although finding a process scenario in which the two very different paradigms are scientifically comparable from a performance perspective will not be easy. Finally, as one of our stated goals is to increase the maturity and acceptance of data-centric process management, we are actively developing advanced capabilities, such as ad-hoc changes and variant support, on top of the presented artifact.

Acknowledgments. This work is part of the ZAFH Intralogistik, funded by the European Regional Development Fund and the Ministry of Science, Research and the Arts of Baden-Wïiœrttemberg, Germany (F.No. 32-7545.24-17/3/1) 


\section{References}

1. Van der Aalst, W.M.P., Weske, M., Grünbauer, D.: Case handling: a new paradigm for business process support. Data \& Knowledge Engineering 53(2), 129-162 (2005)

2. Agha, G., Hewitt, C.: Concurrent programming using actors. In: Readings in Distributed Artificial Intelligence, pp. 398-407. Elsevier (1988)

3. Andrews, K., Steinau, S., Reichert, M.: Enabling fine-grained access control in object-aware process management systems. In: EDOC. pp. 143-152. IEEE (2017)

4. Andrews, K., Steinau, S., Reichert, M.: Towards hyperscale process management. In: Proc EMISA. pp. 148-152 (2017)

5. Bauer, T., Reichert, M., Dadam, P.: Intra-subnet load balancing in distributed workflow management systems. Intl J of Coop Inf Sys 12(03), 295-323 (2003)

6. Cao, J., Jarvis, S.A., Saini, S., Nudd, G.R.: Gridflow: Workflow management for grid computing. In: Intl Symp on Cluster Comp and the Grid. pp. 198-205 (2003)

7. Ceri, S., Grefen, P., Sanchez, G.: WIDE - a distributed architecture for workflow management. In: RIDE-WS. pp. 76-79. IEEE (1997)

8. Cohn, D., Hull, R.: Business artifacts: A data-centric approach to modeling business operations and processes. Bulletin IEEE TCDE 32(3), 3-9 (2009)

9. Duipmans, E.F., Pires, L.F., da Silva Santos, L.O.B.: Towards a bpm cloud architecture with data and activity distribution. In: Enterprise Distributed Object Computing Conference Workshops (EDOCW), 2012 IEEE 16th International. pp. 165-171. IEEE (2012)

10. Euting, S., Janiesch, C., Fischer, R., Tai, S., Weber, I.: Scalable business process execution in the cloud. In: Cloud Engineering (IC2E), 2014 IEEE International Conference on. pp. 175-184. IEEE (2014)

11. Han, Y.B., Sun, J.Y., Wang, G.L., Li, H.F.: A cloud-based bpm architecture with user-end distribution of non-compute-intensive activities and sensitive data. Journal of Computer Science and Technology 25(6), 1157-1167 (2010)

12. Hoefler, T., Belli, R.: Scientific benchmarking of parallel computing systems. In: IEEE SC15. p. 73. ACM (2015)

13. Künzle, V., Reichert, M.: PHILharmonicFlows: towards a framework for objectaware process management. JSME 23(4), 205-244 (2011)

14. Künzle, V., Reichert, M.: Striving for object-aware process support: How existing approaches fit together. In: SIMPDA. pp. 169-188. Springer (2011)

15. Le Boudec, J.Y.: Performance evaluation of computer systems. Epfl Press (2010)

16. Leymann, F., Roller, D.: Production workflow: concepts and techniques. Prentice Hall PTR Upper Saddle River (2000)

17. Schuler, C., Weber, R., Schuldt, H., Schek, H.J.: Scalable peer-to-peer process management - the osiris approach. In: IEEE ICWS. pp. 26-34 (2004)

18. Schulte, S., Janiesch, C., Venugopal, S., Weber, I., Hoenisch, P.: Elastic business process management. FGCS 46, 36-50 (2015)

19. Steinau, S., Andrews, K., Reichert, M.: The Relational Process Structure. In: 30th Int'l Conf. on Advanced Information Systems Engineering (CAiSE). pp. 53-67. Springer (2018)

20. Steinau, S., Künzle, V., Andrews, K., Reichert, M.: Coordinating business processes using semantic relationships. In: Proc CBI. pp. 143-152 (2017) 